\title{
Reply to Martilli et al.: "Summer average urban-rural surface temperature differences do not indicate the need for urban heat reduction"
}

\author{
Gabriele Manoli ${ }^{1}$, Simone Fatichi², Markus Schläpfer ${ }^{3}$, Kailiang Yu ${ }^{4}$, Thomas W. Crowther ${ }^{5}$, Naika \\ Meili $^{3 ; 6}$, Paolo Burlando 6 , Gabriel G. Katul ${ }^{7}$, \& Elie Bou-Zeid ${ }^{8}$ \\ ${ }^{1}$ Department of Civil, Environmental and Geomatic Engineering, University College London, UK \\ ${ }^{2}$ Department of Civil and Environmental Engineering, National University of Singapore, Singapore \\ ${ }^{3}$ Future Cities Laboratory, Singapore-ETH Centre, ETH Zurich, 138602 Singapore \\ ${ }^{4}$ Laboratoire des Sciences du Climat et de l'Environnement, Commissariat à l'Énergie Atomique et aux Énergies \\ Alternatives, CNRS Université de Versailles-Saint-Quentin-en-Yvelines, France \\ ${ }^{5}$ Department of Environmental Systems Science, ETH Zurich, Zurich, Switzerland \\ ${ }^{6}$ Institute of Environmental Engineering, ETH Zurich, Zurich, Switzerland \\ ${ }^{7}$ Nicholas School of the Environment, Duke University, Durham, NC 27708, USA \\ ${ }^{8}$ Department of Civil and Environmental Engineering, Princeton University, Princeton, NJ 08544, USA
}

This is a reply to Martilli et al. (2020), Summer average urban-rural surface temperature differences do not indicate the need for urban heat reduction, https://doi.org/10.31219/osf.io/8gnbf

Martilli et al. question the utility of the coarse-grained model presented in Manoli et al. (2019) and criticize the use of Surface Urban Heat Island (SUHI) intensities as metric for heat risk and mitigation. The authors make two conceptual points: (1) air temperature $\left(T_{a}\right)$ is more important than surface temperature $\left(T_{s}\right)$ for local scale interventions and (2) city-scale averages - and the concept of "urban heat island" itself - are irrelevant due to the large spatio-temporal variability of urban characteristics and the fact that absolute temperatures in cities are more informative than the difference relative to a rural background. While point (1) highlights an important issue - already discussed in our published article - we respectfully disagree on point (2), which is in conflict with the vast literature on SUHIs (to which the authors themselves have largely contributed) and the parallel field of urban complexity. This last point prompts some teleological considerations on current and future directions in urban climate research, as discussed in detail here.

In general, the criticism by Martilli et al. originates from a misinterpretation of the scales and scope of our analysis: while we are aware of the complexity and heterogeneity of cities, the approach featured in the paper intentionally focuses on multi-city scale conditions averaged in time and over a global ensemble of urban areas with similar population and precipitation. That is, the focus remains on emergent global patterns and seasonal averages, a focus that is purposely distinct from most current canonical urban climate studies and parameterizations dealing with block/neighbourhood/single-city scale processes (which are important, but not our focus here). The limitations of such a global scale effort have been acknowledged and discussed in our paper, and inevitably some trade-offs with finescale processes have been made. This is unambiguously stated in the published manuscript, and we have no reason to suspect readers will not be mindful of these limitations when interpreting our conclusions. We specifically state that the findings should not be used for policy making for an individual city or neighbourhood. However, similar coarse-grained global scaling analyses have been critically important in many other fields (e.g. the Budyko curve in hydrology as illustrated, for example, 
by Berghuijs et al. 2014), and yet they remained lacking in urban climatology where they can assist in framing the increasing number of global modelling and observational studies of cities.

We note a typo in the SI of Manoli et al. 2019 and we acknowledge that a few sentences might benefit from some clarifications (see Supplementary Information) to make sure there is no room for misinterpretation by decision makers. However, the results and conclusions of our published article are not affected by any of the issues raised by Martilli et al., they are robust, correct given the scale and aim of the study, and congruent with existing literature on urban climate and city analytics.

Air vs Surface temperature. We do not question "the complexity of the $T_{s}-T_{a}$ relationship", especially when dealing with building/block scale processes at hourly or daily resolutions, and with smaller cities near coastlines. However, the statement that a clear relation between air temperature and remotelysensed surface temperature has not been established at the scale of our analysis is incorrect (e.g. Zhou et al. 2013, Zhou et al. 2019). Our study focuses on global patterns and seasonal or longer timescales and NOT hourly/daily conditions at specific locations where differences between air and surface temperatures could potentially be significant due to the processes mentioned by Martilli et al (which are all weather-scale and not climate-scale processes). On a relatively long-period, $T_{s}$ and $T_{a}$ are tightly connected as can be clearly seen in all global datasets (e.g. Hooker et al. 2018). Our study also focuses on city-to-city variations and not on temporal correlations between $T_{s}$ and $T_{a}$ or $T_{s}$ and the model inputs. This is in line with observations by Zhang et al. (2014b) showing that air and surface temperatures from 300 urban areas in the US exhibit the same spatial patterns.

Regarding urban-rural changes, we are well aware of the difference between air and surface UHIs (e.g. see discussion in Manoli et al. 2020, PNAS) and we specifically focus on surface UHIs (SUHIs). Given the apparent misinterpretation, we should have probably used the acronym SUHI (rather than UHI) throughout the paper. We recognize that we could have better phrased a few sentences to clearly distinguish between air UHI and SUHI (see comments in the Supplementary Information). However, the authors incorrectly state that the two types of UHIs are uncorrelated and mention Fig. $4 \mathrm{f}$ in Zhou et al. 2013 to support their claim. In time and over the course of a diurnal cycle, air UHI and SUHI might indeed be anti-correlated since SUHI peaks during the day while air UHIs generally peak at night, so it is not surprising that the data mentioned by the authors lack correlations as they refer to daytime conditions only (see Zhou et al. 2013). On the other hand, if one considers the spatial (inter-city) correlation of SUHI and air UHI averaged at synoptic time scales or longer, there are strong links as cities and periods with large mean SUHI will experience a large mean air UHI (Sun et al. 2020). In summary, while the relationship between air and surface UHIs is not trivial, on proper timescales, there is compelling evidence showing their correlation (see review by Zhou et al. 2019). For example, Zhang et al. (2014a) derived a simple linear relation linking nocturnal air and surface observations.

Regarding the fact that satellite-derived $T_{s}$ "represents only a subset of urban surfaces seen by the radiometer" and is therefore not fully representative of the 3D urban context, we first underline the fact that longwave emissions from the ground (and to a lesser extent walls) inside the canyon also contribute to the satellite-observed temperature (Li and Bou-Zeid 2014), and the latter is therefore not solely a roof temperature measurement. Moreover, this "lumped" radiant skin temperature is perfectly in line with the assumptions of our model and not inconsistent with it. Our coarse-grained approach intentionally focuses on "city-scale" averages, simplifying the complex 3D structure of cities to few bulk variables. Hence, city-scale values of SUHIs retrieved from remote sensing are the best observations to test and validate the model. The model simulates a "lumped single skin-surface 
temperature", which is the closest variable to what a satellite with a coarse spatial resolution may observe as surface temperature.

We completely agree (and made it clear in the paper) that our approach is not comparable with air temperature inside urban canyons, urban parks, or any other local area, or with the temperature of specific surfaces, but that is not the purpose of our analysis or model. This has been consistently misinterpreted by the authors. We however must underline that, while ideally one would be able to access both $T_{s}$ and $T_{a}$ for analyses (rarely the case), $T_{s}$ on its own has direct implications for thermal comfort (due to radiative loading on pedestrians outdoors) and on building energy consumption (due to heat conduction through building envelope). In fact, for modern well-sealed buildings, $T_{s}$ is a much more direct indicator of cooling load than $T_{a}$ (the latter would only capture convective heat exchange with the environment). One should also note that most urban heat mitigation measures (e.g. green or cool surface) target $T_{s}$ and expect a positive impact on $T_{a}$ as the sensible heat flux is reduced. Even in large-scale models of uniform vegetation, $T_{s}$ may be preferred because $T_{a}$ varies appreciably with height within and above surfaces. Hence, selecting a reference $T_{a}$ to compare against is far more problematic than $T_{s}$ as has been known for quite some time (e.g. Novick and Katul 2020)

UHIs and heat mitigation. We respectfully disagree with the authors on this point. We are keenly aware of the distinction between measures for broadly improving local microclimate (e.g. Meili et al. 2020), versus mitigation measures for reducing the UHI. We are also cognizant of the possible positive implications of UHIs in cold climates in the winter and the potential negative effects of conventional mitigation measures (Yang and Bou-Zeid 2018). However, the local microclimate is not unrelated to the intensity of UHIs as Martilli et al. seem to suggest. Given a constant rural reference, modifying the SUHI modifies the surface, canopy, and boundary layer absolute temperatures over the entire city. Therefore, UHI remains a key indicator of urban climate studies: it measures how better or worse the modified city climate is relative to its background conditions.

The authors themselves have largely contributed to the literature on the topic (e.g. Roth et al. 1989, Connors et al. 2013, Allen et al. 2017, Bechtel et al. 2019a,b). On this issue, we note that Bechtel et al. (2019b) have investigated the seasonal hysteresis of SUHIs using monthly averaged data (i.e. the same spatial and temporal resolution of our global analysis). This hysteretic phenomenon, originally presented elsewhere (Zhou et al. 2013), has been explained only recently (Manoli et al. 2020, PNAS) thanks to a refined version of our coarse-grained model that was shown to capture the SUHI dynamics at monthly time-scales.

We are well aware that pedestrian-level outdoor thermal comfort and the risk of heat-related mortality is linked to short-lived localized episodes rather than longer-term space-time averages. This requires a shift (that we endorse) in the focus from urban climate to urban weather studies, and their interaction with synoptic conditions. Addressing such "fine-scale" processes requires a completely different type of analysis and it was not the aim of our study. To be clear, air temperature itself is not enough to quantify possible impacts on public health as thermal comfort depends on multiple factors (including air humidity and surface temperatures, e.g. Höppe 1999) and risk varies with exposure and vulnerability (e.g. age, socioeconomic conditions, acclimatization) (Gasparrini et al. 2015, Hondula et al. 2018). A single indicator is clearly not enough for such a complex and geographically variable problem and background climate (as well as intra-urban variability) should be surely taken into account when considering potential impacts on city dwellers. Yet, remotely-sensed $T_{s}$ remains a useful and widely-used indicator for spatially-explicit epidemiology studies (Kestens et al., 2011, Laaidi et al., 2012, Venter et al 2020) and city-wide decision making (Schwarz et al. 2012, Chakraborty et al. 2020). 
We do agree that heat mitigation strategies must aim "to reduce the negative effects of urban heat, and not urban-rural temperature differences", but it is important to clarify that cities "cannot control their background climate, they can only influence the urban-induced perturbation from that background" (Manoli et al. 2020, PNAS). Hence, the UHI serves as one measure of what is feasible given the "hand a city is dealt" climatically.

Regarding the comment on green spaces, it is true that our results "do not indicate how $T_{a}$ would change from altering the greening fraction" because it was not the aim of our study - which, we reiterate, focused on SUHIs only. To quantify the effects of vegetation and other mitigation measures on the canyon-scale microclimate - including air temperature - we are developing/using different tools (Meili et al. 2020). We can note, however, that there is a clearly established correlation between the benefits of green or cool infrastructure in reducing air and surface UHIs simultaneously, even at hourly timescales (e.g. Li et al. 2014).

In general, none of the comments by Martilli et al. present any evidence or references that contradict our basic conclusion, i.e. the ability of urban green spaces to reduce the SUHI effect varies strongly with background climate, and is reduced in humid regions. There is a consistent bias towards solutions that focus on urban vegetation at the city scale, with confusion in extrapolating local-scale results to the city level (Yang and Bou-Zeid 2019) and results from some climates to others. We find this bias to be counter-productive. Over prescribing vegetation, green infrastructures and cool roofs as necessarily effective city-scale solutions globally without adequate consideration of the climatic influence is a key concern that motivated the analysis here. Our paper is simply a note of caution based on clear energy budget and thermodynamic considerations. As such, our paper in fact underlines the need for local fine-grained studies when designing particular urban heat mitigation solutions.

Finally, we note that the claim that " $\Delta T_{s}$ does not simply represent an urban perturbation on top of a rural background" is incorrect. It is a well-established practice to describe large scale land use conversion as an external perturbation and derive first-order approximations of surface temperature changes by linearizing the surface energy balance equation (e.g. Lee et al. 2011, Zhao et al. 2014, Bright et al. 2017, Zeng et al. 2017). This is precisely because both urban and rural areas are subjected to the same atmospheric forcing and any "coarse-grained" change in the surface energy balance can be described by changes (or perturbations) in the underlying control parameters (e.g. Lee et al. 2011), as Martilli et al. suggest in their comment in fact. While for urban surfaces the linearity of this perturbation can be debated, it remains a perturbation to the background.

Concluding remarks. The comments by Martilli et al. are interpreting our paper through the lens of classic urban climate studies focusing on building block to neighborhood scale processes, the specifics of single cities, and particular weather events (e.g. heatwaves). These are all extremely relevant aspects of urban microclimate, but they are not the focus of our study. Our study proposes a methodology that inevitably sacrifices such "fine-grain" processes (i.e. those that exist within cities) but offers a way forward in explaining emergent behaviors of human-natural systems at the global scale (i.e. across similar sized cities with differing climate). For the sake of clarity, the definition of emergence is provided in the Supplementary Information. To be clear, many "local-scale" approaches are based on bulk parameterization of finer scales processes, e.g. the use of local climate zones (Demuzere et al. 2019) is nothing but a coarse-grained representation of far more complex urban structures. As a case in point, note that juxtaposition of the criticism by Martilli et al. to global climate change would suggest that mean global surface warming is not a valid metric for assessing climate change trends as the "variability of fine-scale features is too large and too relevant to be neglected" 
for action at the local scale. Yet, only a coarse-grained approach can reveal causal links between global emissions and warming and be used to generalize guidelines for heat mitigation, which of course can be fine-tuned locally to capture "variability of fine-scale features" and background climate.

Clearly, this is not the first instance in science when coarse-grained (i.e. spatially averaged models with all parameters treated as effective) and detailed spatially-explicit approaches (i.e. local models where geometry and small-scale processes are explicitly considered) are debated and criticized. Examples from other disciplines where simplicity versus complexity of models (Paola and Leeder 2011) was debated include watershed hydrology (e.g. Michaud \& Sorooshian, 1994; Bormann et al. 2009), ecology (e.g. Evans et al. 2013), geomorphology (Dietrich et al. 2003), and boundary-layer dynamics (e.g. Bellon \& Stevens 2012). Urban climate research can benefit from such a debate as the criticism by Martilli et al. reveals the power of the "tyranny of small scales" (Or 2019). In our view, urban climate research can substantially benefit from a convergence of approaches and a constructive discussion on what degree of physical approximation is deemed acceptable to tackle local and global challenges. Detailed urban climate approaches are extremely important and their applications cannot be performed by our coarse-grained approach - yet complementing them can offer new ways for upscaling and downscaling complex socio-ecological processes. As prophetically envisioned by Klemeš (1983) for hydrology and recently highlighted for Earth system modeling (Or 2019), also urban climate research can only "jump ahead after its links with processes at the planetary level are better established, in a similar way as advances in chemistry were made possible by developments in atomic physics. This belief stems from an observation that a successful solution of a problem is more likely if it is approached from two opposite directions". In the case of urban climate, research can capitalize on the recent advances in big data, city-analytics and complexity science (e.g. Batty 2008, Bettencourt \& West 2010, Bettencourt et al. 2020) to explore the "downwards" route from global to local concepts and search for "a rigorous and formal reconciliation of scales" (Or 2019).

\section{References}

Allen, M. A., Voogt, J. A., \& Christen, A. (2017). Towards a continuous climatological assessment of urban surface heat islands. In 2017 Joint Urban Remote Sensing Event (JURSE) (pp. 1-4). IEEE.

Batty, M. (2008). The size, scale, and shape of cities. science, 319(5864), 769-771.

Bechtel, B., Demuzere, M., Mills, G., Zhan, W., Sismanidis, P., Small, C., \& Voogt, J. (2019a). SUHI analysis using Local Climate Zones-A comparison of 50 cities. Urban Climate, 28, 100451.

Bechtel, B., Sismanidis, P., Voogt, J., \& Zhan, W. (2019b). Seasonal Surface Urban Heat Island Analysis. In 2019 Joint Urban Remote Sensing Event (JURSE) (pp. 1-4). IEEE.

Bellon, G., \& Stevens, B. (2012). Using the sensitivity of large-eddy simulations to evaluate atmospheric boundary layer models. Journal of the atmospheric sciences, 69(5), 1582-1601.

Berghuijs, W. R., Woods, R. A., \& Hrachowitz, M. (2014). A precipitation shift from snow towards rain leads to a decrease in streamflow. Nature Climate Change, 4(7), 583-586.

Bettencourt, L., \& West, G. (2010). A unified theory of urban living. Nature, 467(7318), 912-913.

Bettencourt, L. M., Yang, V. C., Lobo, J., Kempes, C. P., Rybski, D., \& Hamilton, M. J. (2020). The interpretation of urban scaling analysis in time. Journal of the Royal Society Interface, $17(163)$, 20190846.

Bormann, H., Breuer, L., Giertz, S., Huisman, J. A., \& Viney, N. R. (2009). Spatially explicit versus lumped models in catchment hydrology-experiences from two case studies. In Uncertainties in environmental modelling and consequences for policy making (pp. 3-26). Springer, Dordrecht. 
Bright, R. M., Davin, E., O'Halloran, T., Pongratz, J., Zhao, K., \& Cescatti, A. (2017). Local temperature response to land cover and management change driven by non-radiative processes. Nature Climate Change, 7(4), 296-302.

Chakraborty, T. C., Hsu, A., Manya, D., \& Sheriff, G. (2020). Urban Heat Island Calculations for Decision Making in the United States: Characterization and Implications. eartharxiv.org

Connors, J. P., Galletti, C. S., \& Chow, W. T. (2013). Landscape configuration and urban heat island effects: assessing the relationship between landscape characteristics and land surface temperature in Phoenix, Arizona. Landscape ecology, 28(2), 271-283.

Demuzere, M., Bechtel, B., \& Mills, G. (2019). Global transferability of local climate zone models. Urban climate, 27, 46-63.

Dietrich, W. E., Bellugi, D. G., Sklar, L. S., Stock, J. D., Heimsath, A. M., \& Roering, J. J. (2003). Geomorphic transport laws for predicting landscape form and dynamics. Geophysical Monograph-American Geophysical Union, 135, 103-132.

Evans, M. R., Grimm, V., Johst, K., Knuuttila, T., De Langhe, R., Lessells, C. M., ... \& Wilkinson, D. J. (2013). Do simple models lead to generality in ecology? Trends in ecology \& evolution, 28(10), 578-583.

Gasparrini, A., Guo, Y., Hashizume, M., Lavigne, E., Zanobetti, A., Schwartz, J., ... \& Leone, M. (2015). Mortality risk attributable to high and low ambient temperature: a multicountry observational study. The Lancet, 386(9991), 369-375.

Hondula, D. M., Davis, R. E., \& Georgescu, M. (2018). Clarifying the connections between green space, urban climate, and heat-related mortality.

Hooker, J., Duveiller, G., \& Cescatti, A. (2018). A global dataset of air temperature derived from satellite remote sensing and weather stations. Scientific data, 5(1), 1-11.

Höppe, P. (1999). The physiological equivalent temperature-a universal index for the biometeorological assessment of the thermal environment. International journal of Biometeorology, 43(2), 71-75.

Kestens, Y., Brand, A., Fournier, M., Goudreau, S., Kosatsky, T., Maloley, M., \& Smargiassi, A. (2011). Modelling the variation of land surface temperature as determinant of risk of heat-related health events. International journal of health geographics, 10(1), 7.

Klemeš, V. (1983). Conceptualization and scale in hydrology. Journal of hydrology, 65(1-3), 1-23.

Laaidi, K., Zeghnoun, A., Dousset, B., Bretin, P., Vandentorren, S., Giraudet, E., \& Beaudeau, P. (2012). The impact of heat islands on mortality in Paris during the August 2003 heat wave. Environmental health perspectives, 120(2), 254-259.

Lee, X., Goulden, M. L., Hollinger, D. Y., Barr, A., Black, T. A., Bohrer, G., ... \& Katul, G. (2011). Observed increase in local cooling effect of deforestation at higher latitudes. Nature, 479(7373), 384-387.

Li, D., \& Bou-Zeid, E. (2014). Quality and sensitivity of high-resolution numerical simulation of urban heat islands. Environmental Research Letters, 9(5), 055001.

Li, D., Bou-Zeid, E and Oppenheimer, M (2014). The Effectiveness of Cool and Green Roofs as Urban Heat Island Mitigation Strategies. Environmental Research Letters, 9, 055002.

Manoli, G., Fatichi, S., Schläpfer, M., Yu, K., Crowther, T. W., Meili, N., ... \& Bou-Zeid, E. (2019). Magnitude of urban heat islands largely explained by climate and population. Nature, 573(7772), 55-60.

Manoli, G., Fatichi, S., Bou-Zeid, E., \& Katul, G. G. (2020). Seasonal hysteresis of surface urban heat islands. Proceedings of the National Academy of Sciences, 117(13), 7082-7089. 
Meili, N., Manoli, G., Burlando, P., Bou-Zeid, E., Chow, W. T., Coutts, A. M., ... \& Fatichi, S. (2020). An urban ecohydrological model to quantify the effect of vegetation on urban climate and hydrology (UT\&C v1. 0). Geoscientific Model Development, 13, 335-362.

Michaud, J., \& Sorooshian, S. (1994). Comparison of simple versus complex distributed runoff models on a midsized semiarid watershed. Water resources research, 30(3), 593-605.

Novick, K. A., \& Katul, G. G. (2020). The Duality of Reforestation Impacts on Surface and Air Temperature. Journal of Geophysical Research: Biogeosciences, e2019JG005543.

Or, D. (2019). The tyranny of small scales-on representing soil processes in global land surface models. Water Resources Research.

Paola, C., \& Leeder, M. (2011). Simplicity versus complexity. Nature, 469(7328), 38-39.

Roth, M., Oke, T. R., \& Emery, W. J. (1989). Satellite-derived urban heat islands from three coastal cities and the utilization of such data in urban climatology. International Journal of Remote Sensing, 10(11), 1699-1720.

Schwarz, N., Schlink, U., Franck, U., \& Großmann, K. (2012). Relationship of land surface and air temperatures and its implications for quantifying urban heat island indicators -An application for the city of Leipzig (Germany). Ecological indicators, 18, 693-704.

Sun, T., Sun, R., \& Chen, L. (2020). The Trend Inconsistency between Land Surface Temperature and Near Surface Air Temperature in Assessing Urban Heat Island Effects. Remote Sensing, 12(8), 1271.

Venter, Z. S., Krog, N. H., \& Barton, D. N. (2020). Linking green infrastructure to urban heat and human health risk mitigation in Oslo, Norway. Science of the Total Environment, 709, 136193.

Yang, J., \& Bou-Zeid, E. (2018). Should cities embrace their heat islands as shields from extreme cold? Journal of Applied Meteorology and Climatology, 57(6), 1309-1320.

Yang, J., \& Bou-Zeid, E. (2019). Scale dependence of the benefits and efficiency of green and cool roofs. Landscape and urban planning, 185, 127-140.

Zeng, Z., Piao, S., Li, L. Z., Zhou, L., Ciais, P., Wang, T., ... \& Mao, J. (2017). Climate mitigation from vegetation biophysical feedbacks during the past three decades. Nature Climate Change, $7(6)$, 432-436.

Zhao, L., Lee, X., Smith, R. B., \& Oleson, K. (2014). Strong contributions of local background climate to urban heat islands. Nature, 511(7508), 216-219.

Zhang, F., Cai, X., \& Thornes, J. E. (2014a). Birmingham's air and surface urban heat islands associated with Lamb weather types and cloudless anticyclonic conditions. Progress in physical geography, 38(4), 431-447.

Zhang, P., Bounoua, L., Imhoff, M. L., Wolfe, R. E., \& Thome, K. (2014b). Comparison of MODIS land surface temperature and air temperature over the continental USA meteorological stations. Canadian Journal of Remote Sensing, 40(2), 110-122.

Zhou, B., Rybski, D., \& Kropp, J. P. (2013). On the statistics of urban heat island intensity. Geophysical research letters, 40(20), 5486-5491.

Zhou, D., Xiao, J., Bonafoni, S., Berger, C., Deilami, K., Zhou, Y., ... \& Sobrino, J. (2019). Satellite remote sensing of surface urban heat islands: progress, challenges, and perspectives. Remote Sensing, 11(1), 48. 


\title{
Supplementary Information
}

\section{Reply to Martilli et al.: "Summer average urban-rural surface temperature differences do not indicate the need for urban heat reduction"}

\author{
Gabriele Manoli ${ }^{1}$, Simone Fatichi ${ }^{2}$, Markus Schläpfer ${ }^{3}$, Kailiang Yu ${ }^{4}$, Thomas W. Crowther ${ }^{5}$, Naika \\ Meili $^{3 ; 6}$, Paolo Burlando 6 , Gabriel G. Katul ${ }^{7}$, \& Elie Bou-Zeid ${ }^{8}$ \\ ${ }^{1}$ Department of Civil, Environmental and Geomatic Engineering, University College London, UK \\ ${ }^{2}$ Department of Civil and Environmental Engineering, National University of Singapore, Singapore \\ ${ }^{3}$ Future Cities Laboratory, Singapore-ETH Centre, ETH Zurich, 138602 Singapore \\ ${ }^{4}$ Laboratoire des Sciences du Climat et de l'Environnement, Commissariat à l'Énergie Atomique et aux Énergies \\ Alternatives, CNRS Université de Versailles-Saint-Quentin-en-Yvelines, France \\ ${ }^{5}$ Department of Environmental Systems Science, ETH Zurich, Zurich, Switzerland \\ ${ }^{6}$ Institute of Environmental Engineering, ETH Zurich, Zurich, Switzerland \\ ${ }^{7}$ Nicholas School of the Environment, Duke University, Durham, NC 27708, USA \\ ${ }^{8}$ Department of Civil and Environmental Engineering, Princeton University, Princeton, NJ 08544, USA
}

A detailed response to the comments in the Supporting Information by Martilli et al. is provided below. We also note that there is a typing error in Eq. 24 of the $\mathrm{SI}$ of our published manuscript. The correct parameter values are listed in Table S5 of Manoli et al. (2019), i.e. $a_{Q}=0.057$ (and not 17.42 as written in Eq. 24).

\section{A1. Examples of inaccurate use of the literature}

Martilli et al. have raised concerns about some citations and their relevance for surface versus air UHIs. On this regard, we would like to point out that:

a. The "power law scaling of urban warming with population" has been observed also for surface UHIs (e.g. Clinton and Gong 2013, Zhao et al. 2014) so the sentence highlighted by the authors is not inconsistent with the following discussion on SUHIs;

b. The reference to Oke 1973 was used here as an example of "empirical analyses" in urban climate studies so it is irrelevant whether it refers to air or surface UHIs;

c. The reference to Scott et al. (2018) was intended to support the theory of an "opposite correlation" observed for both air and surface temperatures (hence the two references);

d. The references to Oke 1982 and Shashua-Bar et al 2009 were used to justify the term "oasiseffect" (which is observed from both air and surface data) and mention an example in hot-dry climates;

e. We used the relation by Oke 1973 in Fig. S4 for a qualitative comparison only. We agree that our model is not directly comparable to canopy layer air UHIs but with disagree on that fact that is not representative of "clear sky conditions" - remotely sensed surface temperature $T_{s}$ is based on clear-sky observations. In any case, the objective here was to show the similar behavior observed for both air and surface UHIs. 
In conclusion, while we agree that some details might have been omitted due to the concise writing style of the journal, we disagree that the use of the literature is inaccurate. The distinctions between SUHI and air UHI are self-evident and do not require elaboration.

\section{A2. Other inaccurate assumptions}

a) CIESIN Dataset. The mechanistic model is not influenced by the choice of the urban polygons. The model is anchored to an energy budget and thermodynamic considerations, which are different in the tropics and in semi-arid environments, and is not hinged on any specific dataset. Having a larger or smaller extent of natural surfaces in the urban areas of a database, would simply weaken or amplify the observed SUHI effect, thus requiring some adjustments in the model parameterization. This, however, would not alter in any way the model formulation, results, and conclusion of our study. More specifically:

- we are aware of the uncertainties associated with the CIESIN database. In such large datasets there are always some cities where data are unreliable (e.g. the case of Matera), but there is plenty of counterexamples where boundaries/observations are correct (e.g. Paris, see Figure A1 below). Our analysis focused on global averages rather than specific cities, precisely for this reason. In the Methods section we stated that "The use of multiple data sources introduces uncertainties because of possible discrepancies in methodology and/or urban boundaries. However, this study intentionally focuses on global averages rather than cityspecific conditions so that random biases across cities and climates are minimized". Perhaps this qualifying statement was overlooked.

- It is true that the GRUMP dataset tends to overestimate the urban extents when compared to other global maps of urban areas (e.g. Schneider et al. 2009; Schneider et al. 2010). This is due to the fact that GRUMP relies on buffered census data, thus resulting in a map that corresponds more closely to population than built-up areas (Schneider et al. 2010), which is consistent with our focus on metropolitan areas where the resident population lives rather than administrative city boundaries. This is explicitly articulated in the Methods section of our published article: "the focus is on urban agglomerates, which may include satellite cities and towns".

- Our analysis focuses on global trends/patterns so, even if the GRUMP dataset overestimates the global extent of urban areas, the overestimated definition of cities is consistent across climatic gradients and there is no systematic bias across cities or climates - which is the key point here. The fact that a specific database can overestimate or underestimate the urban extents, could influence the observed magnitude of the SUHIs, but it does not affect the general trends observed at the global scale.

- More in general, we respectfully disagree on the existence of "correct" urban areas different from the ones employed in our study. The definition of city boundaries is an open problem in the science of cities (e.g. Arcaute et al. 2015, Oliveira et al. 2108) and the CIESIN dataset simply provides consistent and homogenized data of population and SUHI intensities at the global scale.

To further clarify these points, we have compared the CIESIN dataset used in our study with SUHI observations presented by Chackraborty and Lee (2019). The results are presented in Figure $\mathbf{A} \mathbf{1}$ and Table A1. The two datasets differ in methodology, period of observations, and city boundaries but 
daytime and nighttime values of SUHIs show similar patterns, despite some obvious discrepancies on the exact values. This provides additional evidence of the validity of our analysis.

(a) Matera

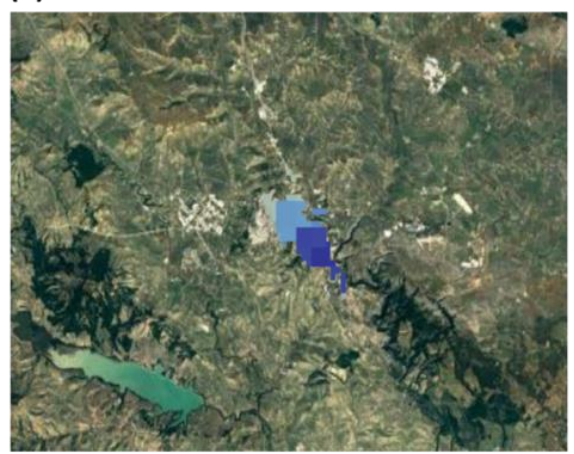

(c)

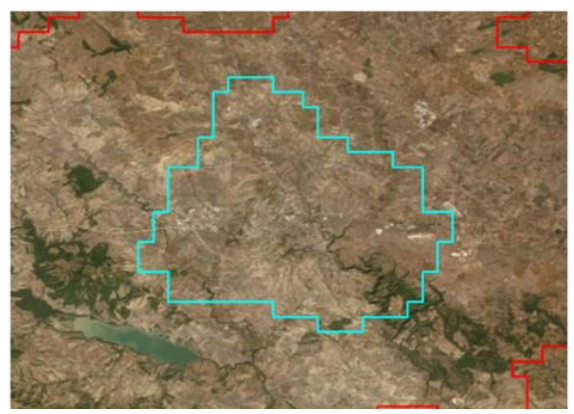

(b) Paris

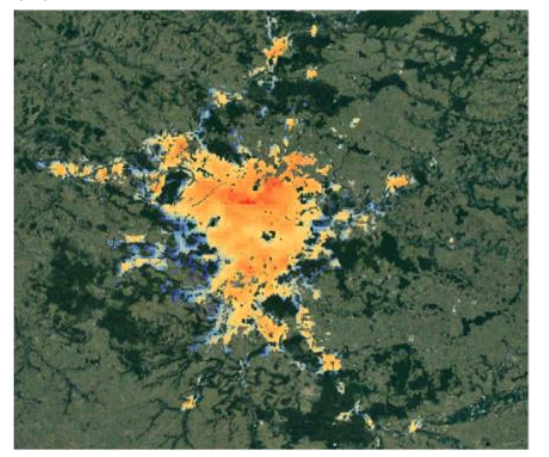

(d)

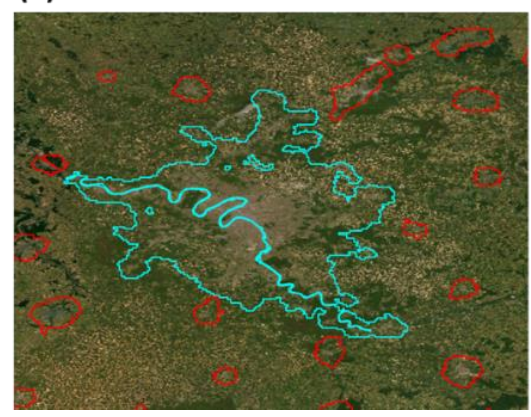

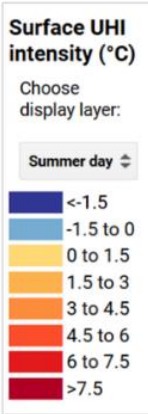

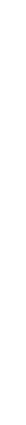

Figure A1: City boundaries for $(a, c)$ Matera and (b,d) Paris as considered by (a-b) Chakraborty and Lee (2019) and (c-d) CIESIN. Note that the two maps employ different projections.

Table A1: UHI intensities during summertime for selected cities. The CIESIN dataset employed in our study is compared with observations by Chackraborty and Lee (2019). Note that the two datasets differ in methodology, city boundaries, and periods of observation.

\begin{tabular}{|l|c|c|c|c|}
\hline & \multicolumn{2}{|c|}{ CIESIN } & \multicolumn{2}{c|}{ Chackraborty and Lee (2019) } \\
\hline City & Summertime UHI intensity $\left[{ }^{\circ} \mathrm{C}\right]$ & \multicolumn{2}{c|}{ Summertime UHI intensity $\left[^{\circ} \mathrm{C}\right]$} \\
\hline & Daytime & nighttime & daytime & nighttime \\
\hline Matera & -0.49 & 0.09 & -0.23 & 1.51 \\
\hline Paris & 2.64 & 1.47 & 2.20 & 1.43 \\
\hline London & 2.64 & 1.01 & 1.77 & 0.85 \\
\hline Teheran & -0.18 & 2.10 & -0.27 & 3.24 \\
\hline Minneapolis & 2.03 & 1.67 & 2.05 & 0.50 \\
\hline
\end{tabular}

b) Definition of $\Delta T_{s}$. We are aware that the CIESIN dataset employs maximum daytime land surface temperature (LST) and minimum nighttime LST during summer. The misunderstanding here is on the real focus and objective of our study: our model/analysis focuses on "climatic patterns" (across cities and climates) and "long-term averages (i.e. daytime/nighttime conditions are smoothed over on seasonal timescale)" (see published article) and not on specific cities, times of day, or weather events. Hence, our study focuses on city-scale conditions averaged over a global ensemble of cities with similar population and precipitation and the choice of "averaged SUHIs" is consistent with such a scope. More in general, our study relies on energy budget and thermodynamics considerations and 
the CIESIN database is only used to confirm global patterns and tune some of the model parameters. In addition, given the diurnal cycle of SUHIs (e.g. Oke et al. 2017), observations at 1:30 am and 1:30 $\mathrm{pm}$ are considered good estimates of summer day and nighttime conditions, respectively (Clinton and Gong 2013) and whether or not they exactly represent the maximum/minimum values of the LST diurnal cycle is a minute detail that is irrelevant for the purpose of our model validation and analysis. Hence, our results/conclusions are not affected by the choice of data and averaging procedure. The trends/observations presented in our manuscript are confirmed also for daytime values (see Figure S3 and Figure S19 in the SI of the published article) and are consistent with other data presented in the literature (e.g. see SI of the published article and reply to the previous comment). Recently, our coarse-grained approach (with some developments for soil moisture) has been successfully employed to describe seasonal dynamics of SUHIs retrieved from different sources (Manoli et al. 2020, PNAS), further confirming the validity of our modeling framework.

c) Urban scaling laws. The authors cite one study (Depersin \& Barthelemy, 2018) to criticize our use of urban scaling relationships. First of all, the study by Depersin \& Barthelemy (2018) focuses on congestion-induced traffic delays and not urban infrastructure size (e.g. area, building height) as more relevant for our model. Second, Depersin \& Barthelemy (2018) addresses "the implication of such scaling forms on individual cities and how they can be used for predicting the behavior of a city when its population changes" which is exactly the opposite of our scope. We agree with the authors that urban scaling laws cannot be applied to extrapolate the characteristics of single cities at a specific moment in time as city-to-city variability can be very large and scaling laws illustrate emergent behaviors of urbanization (i.e. a large ensembles of cities) and not site-specific characteristics. By definition, emergence occurs when a complex system shows large-scale properties that are not necessarily observed at the microscopic level when considering the different system's components (e.g. Kivelson \& Kivelson, 2016). The limitations of scaling laws are discussed elsewhere and not repeated here (Rybski et al. 2019, Arcaute et al. 2020). It is true that scaling values are not "universal" as they depend on the way cities are constructed from data (Arcaute et al. 2015, Cottineau et al. 2017) and how they are compared, e.g. temporal vs cross-sectoral approaches (Bettencourt et al. 2020), but there is a large consensus on the existence of such general relationship (e.g. Li et al. 2017, Bettencourt et al. 2020) and their usefulness for urban studies (e.g. Ribero et al. 2019, Mackenzie et al. 2019).

Regarding the comment on mean building heights, Eq. 14 in the Supplementary Information of our original article has been derived from population and building height data for metropolitan areas in the US (Schläpfer et al. 2015, see below for clarification). The study has been extended to 50 cities in the US, clearly confirming the scaling (Figure A2). A similar relation was also found for UK cities (Molinero and Thurner, 2019). Of course, this is a "coarse-grain" approximation and significant deviations exist when considering one specific city. Nevertheless, it is undeniable that larger cities tend to have higher buildings, and - for the purpose of our study - Eq. 14 is a reasonable first approximation for such changes in mean building height with city population globally. We agree that Eq. 14 ignores the heterogeneities of individual cities but, as explicitly stated in the published article and repeatedly misinterpreted by Martilli et al., our coarse-grained approach focuses on city-wide averages and global scale "collective phenomena and climatic patterns rather than microscopic (i.e., building to block scale) processes", so that our choice of "city-averaged" parameters is justified. Note that similar bulk parameterizations are common assumptions in reduced analytical models (e.g. Li and Bou-Zeid 2013) and slab representations (e.g. Theeuwes et al., 2015) of the urban boundary layer. 
Regarding the data illustrated by Martilli et al. (Figure S3), it is important to clarify that: (i) for the North American cities (red symbols) Martilli et al. mistakenly used the raw data presented in Schläpfer et al. (2015). These raw data are based on different underlying areas (leading to biased samples) and can therefore not be taken for testing the scaling relationship used in our original article (note that the authors of Schläpfer et al. (2015) further delineate the urban areas to make the data comparable and to derive the scaling parameters shown in their Fig. $2 \mathrm{~d}$, which are clearly consistent with the ones used in Eq. 14 in the Supplementary Information of our original article, as well as with theoretical arguments of urban scaling theory); (ii) as far as we can judge based on the information at hand, the black symbols in Figure S3 of Martilli et al. are based on urban footprints that are different from the ones used by Schläpfer et al. (2015) and therefore not directly comparable; and (iii) despite obvious differences among cities, also the additional European data by Martilli et al. show a clear positive relation between population and mean building height, which further consolidates the existence of a general scaling relationship. Finally, it is important to point out that, due to the aforementioned limitations, the data presented by Martilli et al. show some clear inconsistencies (e.g. the mean building height of Madrid is much higher than New York city).
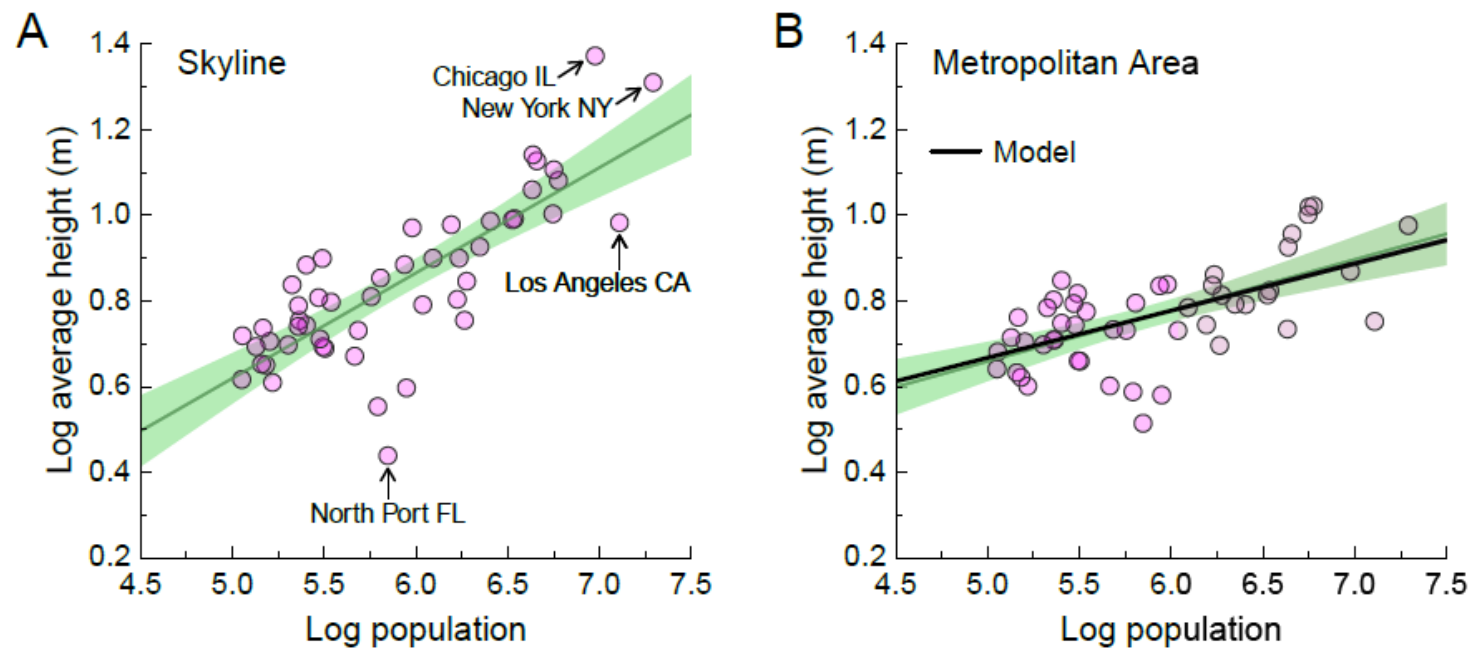

Figure A2: Scaling of mean building height with city size considering (A) city centers and (B) metropolitan areas (Schläpfer et al. 2015, extended to 50 cities). Eq. 14 in the SI of our original article was derived by fitting the data in panel $\mathrm{B}$.

d) Control volume. Martilli et al. must have noted that Eq. 1 is solved at steady state so the storage on the left-hand side is set to zero and both the time derivative and the heat capacity $\mathrm{C}$ are irrelevant to the computations and the model development.

In all cases, to clarify, the energy balance is written considering a lumped urban surface which is in line with, for example, the widely used SEUWS model (Ward et al., 2016). However, the control volume here excludes building mass and considers only an interfacial skin layer with a finite depth (> building height) layer of air above it. Hence, we formulated the storage with $C_{\text {air }}$ since the interfacial skin part is nominally of infinitesimal depth. The $\underline{T}_{\underline{s}}$ in the storage term and the other terms on the right hand side is a lumped temperature as we mentioned before: it is the effective temperature that drives exchanges of heat between this control volume and the air above the canopy. This includes the 
upwelling radiation that is measured by satellites. In this framework, the anthropogenic heat is added since it is a direct source inside our control volume that heats this "urban skin". By increasing the true air temperature near the surface, $Q_{q h}$, reduces the convective cooling of the walls/roofs/ground and of our control volume and increases both the true skin temperature and our effective lumped temperature.

Thus, Eq. 1 in the Supplementary Information of Manoli et al. (2019) correctly describes our modeling framework.

e) Specific city characteristics. The uncertainties related to various confounding effects (e.g. the distance from the coast and elevation), have been clearly mentioned in the manuscript. Given our objective of investigating emergent behaviors at the global scale, we intentionally analyzed all the city available in the dataset. It is true that our results may not hold for specific cities, just like the temperature of a gas does not describe the kinetic energy of each gas molecule/atom individually. However, (i) the peculiarities of specific cities go well beyond elevation and distance from the coast (e.g. presence of rivers, lakes, topography, shape/directionality of the urban area) and introducing all these filters would have limited the scope and generality of our analysis and (ii) most of these factors have a direct impact on air temperature (which is not the focus of our analysis, as mistakenly interpreted by the authors) rather than surface conditions (the objective of our study). As unambiguously stated in the manuscript, the aim of our study is to identify and explain emergent behaviors of global urban-rural systems, which requires a large ensemble of cities to average out the effect of local peculiarities (see previous comment). Recent work has demonstrated that our "coarsegrained" approach, when properly tuned locally, can still provide information for selected cities, including London (Manoli et al. 2020, PNAS), but the global scale results in Manoli et al. (2019) cannot be directly translated into conclusions for a specific city where site characteristics might play a "dominant role in regulating the SUHI". This was clearly stated in our published article.

\section{References}

Arcaute, E., Hatna, E., Ferguson, P., Youn, H., Johansson, A., \& Batty, M. (2015). Constructing cities, deconstructing scaling laws. Journal of The Royal Society Interface, 12(102), 20140745.

Arcaute, E., \& Hatna, E. (2020). Scaling laws: insights and limitations. In Theories and models of urbanization (pp. 45-66). Springer, Cham.

Bettencourt, L. M., Yang, V. C., Lobo, J., Kempes, C. P., Rybski, D., \& Hamilton, M. J. (2020). The interpretation of urban scaling analysis in time. Journal of the Royal Society Interface, 17(163), 20190846.

Chakraborty, T., \& Lee, X. (2019). A simplified urban-extent algorithm to characterize surface urban heat islands on a global scale and examine vegetation control on their spatiotemporal variability. International Journal of Applied Earth Observation and Geoinformation, 74, 269-280.

Clinton, N., \& Gong, P. (2013). MODIS detected surface urban heat islands and sinks: Global locations and controls. Remote Sensing of Environment, 134, 294-304.

Cottineau, C., Hatna, E., Arcaute, E., \& Batty, M. (2017). Diverse cities or the systematic paradox of Urban Scaling Laws. Computers, environment and urban systems, 63, 80-94.

Depersin, J., \& Barthelemy, M. (2018). From global scaling to the dynamics of individual cities. Proceedings of the National Academy of Sciences, 115(10), 2317-2322.

Kivelson, S., \& Kivelson, S. A. (2016). Defining emergence in physics. npj Quantum Materials, 1(1), 12. 
Li, D., \& Bou-Zeid, E. (2013). Synergistic interactions between urban heat islands and heat waves: The impact in cities is larger than the sum of its parts. Journal of Applied Meteorology and Climatology, 52(9), 2051-2064.

Li, R., Dong, L., Zhang, J., Wang, X., Wang, W. X., Di, Z., \& Stanley, H. E. (2017). Simple spatial scaling rules behind complex cities. Nature communications, 8(1), 1-7.

MacKenzie, A. R., Whyatt, J. D., Barnes, M. J., Davies, G., \& Hewitt, C. N. (2019). Urban form strongly mediates the allometric scaling of airshed pollution concentrations. Environmental Research Letters, 14(12), 124078.

Manoli, G., Fatichi, S., Schläpfer, M., Yu, K., Crowther, T. W., Meili, N., ... \& Bou-Zeid, E. (2019). Magnitude of urban heat islands largely explained by climate and population. Nature, 573(7772), 55-60.

Manoli, G., Fatichi, S., Bou-Zeid, E., \& Katul, G. G. (2020). Seasonal hysteresis of surface urban heat islands. Proceedings of the National Academy of Sciences, 117(13), 7082-7089.

Molinero, C., \& Thurner, S. (2019). How the geometry of cities explains urban scaling laws and determines their exponents. arXiv preprint arXiv:1908.07470.

Oke, T. R. (1973). City size and the urban heat island. Atmospheric Environment (1967), 7(8), 769-779.

Oke, T. R. (1982). The energetic basis of the urban heat island. Quarterly Journal of the Royal Meteorological Society, 108(455), 1-24.

Oke, T. R., Mills, G., Christen, A., \& Voogt, J. A. (2017). Urban climates. Cambridge University Press.

Oliveira, E. A., Furtado, V., Andrade, J. S., \& Makse, H. A. (2018). A worldwide model for boundaries of urban settlements. Royal Society open science, 5(5), 180468.

Ribeiro, H. V., Rybski, D., \& Kropp, J. P. (2019). Effects of changing population or density on urban carbon dioxide emissions. Nature communications, 10(1), 1-9.

Rybski, D., Arcaute, E., \& Batty, M. (2019). Urban scaling laws. Environment and Planning B: Urban Analytics and City Science, 1605-1610

Schläpfer, M., Lee, J., \& Bettencourt, L. (2015). Urban Skylines: building heights and shapes as measures of city size. arXiv preprint arXiv:1512.00946. A revised version of this manuscript is in preparation.

Schneider, A., Friedl, M. A., \& Potere, D. (2009). A new map of global urban extent from MODIS satellite data. Environmental Research Letters, 4(4), 044003.

Schneider, A., Friedl, M. A., \& Potere, D. (2010). Mapping global urban areas using MODIS 500-m data: New methods and datasets based on 'urban ecoregions'. Remote Sensing of Environment, 114(8), 1733-1746.

Scott, A. A., Waugh, D. W., \& Zaitchik, B. F. (2018). Reduced Urban Heat Island intensity under warmer conditions. Environmental Research Letters, 13(6), 064003.

Shashua-Bar, L., Pearlmutter, D., \& Erell, E. (2009). The cooling efficiency of urban landscape strategies in a hot dry climate. Landscape and Urban Planning, 92(3-4), 179-186.

Theeuwes, N. E., Steeneveld, G. J., Ronda, R. J., Rotach, M. W., \& Holtslag, A. A. (2015). Cool city mornings by urban heat. Environmental Research Letters, 10(11), 114022.

Ward, H. C., Kotthaus, S., Järvi, L., \& Grimmond, C. S. B. (2016). Surface Urban Energy and Water Balance Scheme (SUEWS): development and evaluation at two UK sites. Urban Climate, 18, 132. 